Benjamin J. Murdock, $\mathrm{PhD}^{*}$

Diane E. Bender, PhD*

Samy R. Kashlan, MD

Claudia Figueroa-

Romero, $\mathrm{PhD}$

Carey Backus, BS

Brian C. Callaghan, MD

Stephen A. Goutman, MD

Eva L. Feldman, MD, $\mathrm{PhD}$

Correspondence to

Dr. Feldman:

efeldman@umich.edu

Supplemental data at Neurology.org/nn

\section{Increased ratio of circulating neutrophils to monocytes in amyotrophic lateral sclerosis}

\section{OPEN}

\section{ABSTRACT}

Objective: To elucidate amyotrophic lateral sclerosis (ALS) biomarkers and potential mechanisms of disease, we measured immune cell populations in whole blood from a large cohort of patients with ALS.

Methods: Leukocytes were isolated from the blood of 44 control patients and 90 patients with ALS. The percentages and total numbers of each cell population were analyzed using flow cytometry and matched with patient ALS Functional Rating Scale-Revised (ALSFRS-R) score to correlate leukocyte metrics with disease progression.

Results: We show a significant increase in the percentage of neutrophils and a significant decrease in the percentage of CD4 T cells and CD16- monocytes in the blood of patients with ALS compared to controls; however, only CD16- monocyte levels correlated with disease progression. We also examined the monocyte surface expression of CCRL2 and CCR3; CD16monocytes displayed decreased percentages and total numbers expressing CCR3, but these numbers did not correlate with ALSFRS-R score. We found that combining multiple disease metrics yielded the most accurate predictor of disease progression: the ratio of neutrophils to CD16- monocytes (N:M ratio) is significantly increased in patients with ALS and better correlates with disease progression than any other single metric.

Conclusions: These observations implicate neutrophils and monocytes as important factors in late disease progression. Neurol Neuroimmunol Neuroinflamm 2016;3:e242; doi: 10.1212/ NXI.0000000000000242

\section{GLOSSARY}

ALS = amyotrophic lateral sclerosis; ALSFRS-R = Amyotrophic Lateral Sclerosis Functional Rating Scale-Revised; CCR = chemokine receptor; $\mathbf{N}: \mathbf{M}$ ratio = neutrophil to $\mathrm{CD}^{1} 6^{-}$monocyte ratio; $\mathbf{S S C}=$ side scatter.

Sporadic amyotrophic lateral sclerosis (ALS) may arise from a myriad of environmental and genetic sources. Neuroinflammation is universally observed in ALS, suggesting all ALS forms ultimately funnel through the immune system, thereby making it an incredibly attractive source of biomarkers and potential therapies. ${ }^{1}$ The immune response in ALS, however, is incredibly complex. This is attributable to the phased nature of the immune system during ALS pathology: the early immune response is dominated by protective cytokines and immune polarization, while the later response is destructive and correlates with rapid disease progression. ${ }^{2}$ Different immune cell types may have a dominant role during each phase, or the same cell type may have opposite roles based on the immune environment. ${ }^{1-3}$ Thus, there is a critical need to elucidate immune mechanisms of ALS and identify potential biomarkers.

In the present study, we phenotyped immune cells in the peripheral blood of patients with ALS and healthy controls to identify immune cell populations linked with ALS progression. We show that several cell populations in patients with ALS are altered relative to healthy controls

\footnotetext{
*These authors contributed equally to this work.

From the Department of Neurology (B.J.M., D.E.B., S.R.K., C.F.-R., C.B., B.C.C., S.A.G., E.L.F.) and A. Alfred Taubman Medical Research Institute (E.L.F.), University of Michigan, Ann Arbor.

Funding information and disclosures are provided at the end of the article. Go to Neurology.org/nn for full disclosure forms. The Article Processing Charge was paid by the authors.

This is an open access article distributed under the terms of the Creative Commons Attribution-NonCommercial-NoDerivatives License 4.0 (CC BY-NC-ND), which permits downloading and sharing the work provided it is properly cited. The work cannot be changed in any way or used commercially.
} 
and that these alterations correlate with disease progression. In particular, we found that the percentage and number of $\mathrm{CD}^{-} 6^{-}$monocytes, as well as the rate of chemokine receptor 3 (CCR3) and CCRL $2^{5,6}$ surface marker expression, are reduced in ALS. In addition, we found that the neutrophil to $\mathrm{CD}_{16}{ }^{-}$monocyte ratio (N:M ratio) correlates best with disease progression.

METHODS Study population. The study population included patients with ALS and healthy controls recruited under an approved protocol (University of Michigan Medical School Institutional Review Board [HUM00028826]). All patients provided written informed consent. Patients with ALS were diagnosed with "probable" or "definite" ALS using the El Escorial World Federation of Neurology criteria ${ }^{7}$ at the University of Michigan ALS Clinic between March 2011 and May 2014. Disease progression was monitored in patients with ALS using the ALS Functional Rating ScaleRevised (ALSFRS-R) in which lower scores correlate with increasing functional impairment. Healthy control patients were recruited via UMClinicalStudies.org; patients with a family history of ALS, previous diagnosis of a neurodegenerative disorder, chronic inflammatory disease, collagen vascular disease, use of immunomodulatory medication, or any fever or sickness at the time of blood draw were excluded.

Standard protocol approvals, registrations, and patient consents. Patients with ALS and healthy controls were consented by the principal investigator or designee using a comprehensive written consent. In brief, the study was reviewed in detail to each patient, patient questions were answered to their satisfaction, consent was reviewed, and the consent form was signed by the patient and principal investigator (or designee).

Blood sample collection and flow cytometry analyses. Blood samples were obtained from patients with ALS and healthy controls by peripheral venipuncture into a $\mathrm{BD}$ Vacutainer sodium heparin tube (BD Biosciences, San Jose, $\mathrm{CA}$ ), placed at $4^{\circ} \mathrm{C}$, and processed within 6 hours of collection. A $200-\mu \mathrm{L}$ aliquot of whole blood was lysed with a red blood cell lysing buffer $(0.8 \%$ $\mathrm{NH}_{4} \mathrm{Cl}, 0.098 \% \mathrm{KHCO}_{3}, 0.1 \mathrm{mM}$ EDTA, and $13.8 \mathrm{mM}$ HEPES), washed with FACS buffer (phosphate-buffered saline, $4 \%$ fetal bovine serum, and $0.1 \%$ sodium azide), and kept on ice throughout the processing and in darkness throughout immunostaining. Cells were counted and incubated in human TruStain FcX block (BioLegend, San Diego, CA) before staining.

\begin{tabular}{|lll|}
\hline Table $1 \quad$ Study population demographics \\
Characteristics & Healthy controls & Patients with ALS \\
No. of patients & 44 & 90 \\
Age range, $y$ & $46-76$ & $38-92$ \\
Age, $y$, mean \pm SD & $59.20 \pm 7.64$ & $61.99 \pm 11.67$ \\
Males, $n$ & 21 & 53 \\
Females, $n$ & 23 & 37 \\
ALSFRS-R score, mean \pm SD & NA & $30.96 \pm 7.48$ \\
ALSFRS-R range & NA & $11-44$ \\
\hline
\end{tabular}

Abbreviations: ALS = amyotrophic lateral sclerosis; ALSFRS-R = Amyotrophic Lateral Sclerosis Functional Rating Scale-Revised; NA = not applicable.
For flow cytometry, a predefined amount of specific monoclonal fluorochrome-labeled antibodies was added to the appropriate tubes and incubated. Flow cytometry was then performed immediately or the cells were fixed with BD Cytofix/Cytoperm for analysis within 48 hours. Phenotyping was performed on a BD FACSCanto II cytometer (BD Biosciences), with voltages set in FACSDiva software 6.1.1. using BD calibration beads (BD CaliBRITE 3; BD Biosciences), while compensations were determined using single color stained and unstained cells. Isotype control gates were set at $\leq 1.0 \%$.

Fluorochrome-conjugated antibodies, including mouse antihuman CD3-FITC (UCHT1), CD4-PerCP (SK3), CD8-APC (SK1), CD14-APC Cy7 (МфP9), CD15-PE Cy5 (H198), CD16-PE (3G8), HLA-DR, DP, DQ-FITC (Tu39), and immunoglobulin isotype-matched control antibodies were purchased from BD. Fluorochrome-conjugated mouse anti-human CCR3PE (5E8), CCRL2-PE (K097F7), CD16-AlexaFluor 647 (3G8), CD8-APC-Cy7 (HIT8a), and immunoglobulin isotype-matched control antibodies were purchased from BioLegend. Initial gating was based on forward vs side scatter (SSC). Subsets for each population were then drawn as follows: the neutrophil gate was drawn from SSC-high population vs CD15 followed by CD16 expression to eliminate eosinophils. Monocyte gates were drawn from the SSC-low population followed by SSC vs CD14; subpopulations were identified using CD16. T cell gates were drawn from SSC vs CD3 followed by CD4 vs CD8. Total numbers of each cell population were determined by multiplying the percentage of each cell type with the total number of leukocytes.

Statistical analyses. Power analysis indicated a sample size of 60 with an $\alpha$ value of 0.05 , and $p=0.5$ provides an estimated power of 0.87958. Statistical analyses and graphing were performed using GraphPad Prism 6.0 (GraphPad Software, Inc., La Jolla, CA). Comparisons between groups were performed using a Student 2-tailed $t$ test utilizing a Bonferroni correction for multiple comparisons; all data are presented as mean \pm SEM. Correlations between immune variables and ALSFRS-R score were expressed using regression analysis in Prism with $R^{2}$ and $p$ values displayed. A value of $p<$ 0.05 was considered statistically significant. Further statistical analyses of the CCR subsets CCRL2 and CCR 3 on CD16 ${ }^{+}$and $\mathrm{CD} 16^{-}$monocytes were performed on a subgroup of the patients in the study; for these analyses concerning CCR3 and CCRL2, extreme outliers were removed using robust regression and outlier removal with a $Q$ coefficient of 1 .

RESULTS Peripheral blood leukocyte levels are altered in patients with ALS. Forty-four healthy control patients (23 women and $21 \mathrm{men}$ ) ranging in age from 46 to 76 years (mean 59.20 years) and 90 patients with ALS (37 women and 53 men) ranging in age from 38 to 92 years (mean 61.99 years) were enrolled in the study (table 1). Following isolation from peripheral blood, leukocytes from each patient were counted, stained with fluorescently labeled antibodies, and analyzed using flow cytometry. Based on cell surface marker expression, we identified neutrophils, $\mathrm{CD}_{16}{ }^{+}$monocytes, $\mathrm{CD} 16^{-}$ monocytes, CD4 T cells, and CD8 T cells in the peripheral blood. The total number of blood leukocytes was not significantly different between patients with ALS and healthy human controls (figure $1 \mathrm{~A}$ ). We found that the percentage of neutrophils within the leukocyte population, but not the total number, was increased in patients 
Figure 1 Immune cell populations are altered in the peripheral blood of patients with ALS

A Total leukocytes

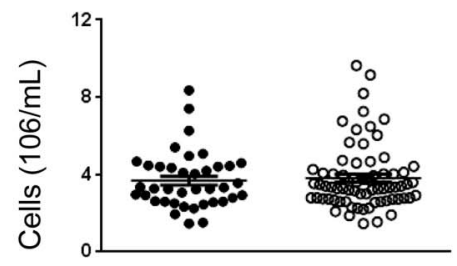

B $\%$ of leukocytes

\# of leukocytes

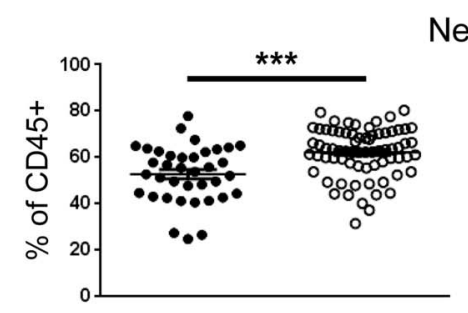

Neutrophils
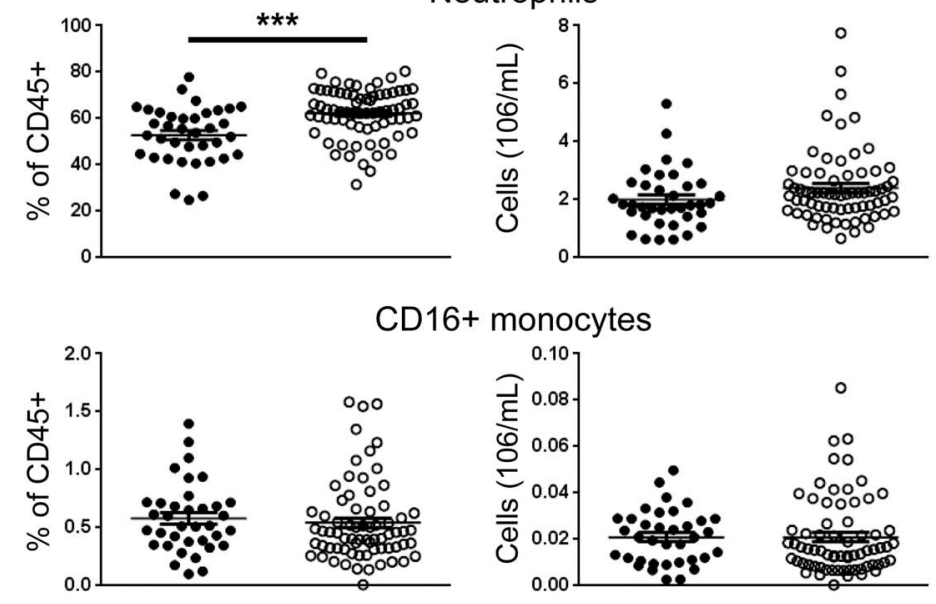

+ monocytes
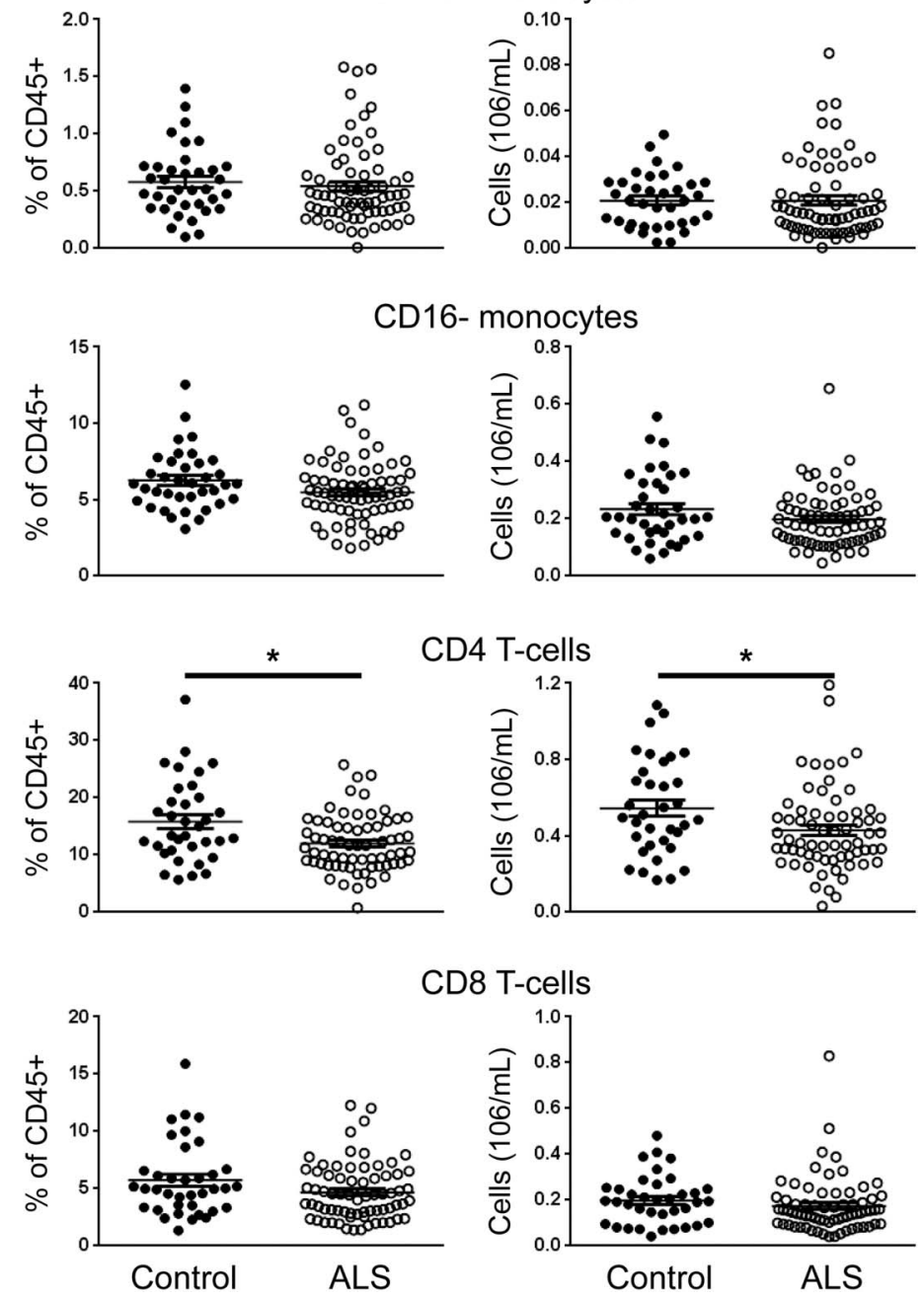

CD8 T-cells

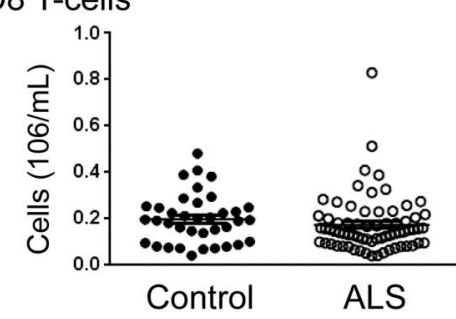

(A) The total number of leukocytes and (B) the percent and total number of specific leukocyte populations are shown. Filled black circles represent individual healthy human control patients, and empty circles represent patients with ALS. Average and SEM are displayed. Asterisks above bars indicate significance $\left(\%\right.$ of $\mathrm{CD}_{4}{ }^{+}$cells: $* p<0.01, * * * p<0.0001$; \# of leukocytes: $* p<$ 0.008). ALS = amyotrophic lateral sclerosis. with ALS relative to healthy controls (figure 1B). Levels of $\mathrm{CD} 16^{+}$monocytes were unaffected in patients with ALS, and we observed a trend toward a lower percentage of blood-borne $\mathrm{CD}_{16}{ }^{-}$monocytes and fewer total $\mathrm{CD} 16^{-}$monocytes, although numbers observed were not significantly different from healthy control patients (figure 1B). Similarly, we observed a significant decrease in the percentage and total number of CD4 T cells, although the levels of CD8 $\mathrm{T}$ cells were not altered in patients with ALS.

Percentages and total number of each cell population were then compared with patient ALSFRS-R score to determine positive or negative correlations between cell types and severity of disease. Total number of leukocytes showed no relationship to the ALSFRS-R score (data not shown). We observed that the percentage and total number of neutrophils both increased as disease progressed, but the relationship did not reach statistical significance, indicating a lack of correlation between the 2 metrics of disease (figure 2). Similarly, we saw no correlation between $\mathrm{CD} 16^{+}$ monocytes or CD8 $\mathrm{T}$ cells and patient ALSFRS-R scores. However, we observed a significant reduction in the percentage of $\mathrm{CD}^{-} 6^{-}$monocytes within the total leukocyte population, although total numbers of $\mathrm{CD}_{16} 6^{-}$monocytes did not correlate with disease progression. Of note, despite the reduced percentage and number of CD4 $\mathrm{T}$ cells observed in the blood of patients with ALS (figure 1B), there was no correlation between ALSFRS-R score and either of these metrics, suggesting that CD4 T cell levels in the blood are reduced at all stages of disease.

CCR3 and CCRL2 monocyte surface expression is altered during ALS. Based on these findings, we wished to examine whether surface markers on $\mathrm{CD}^{-} 6^{-}$monocytes were altered during the course of ALS. We have previously shown that markers that are expressed by monocytes are upregulated in the CNS of patients with ALS, particularly CCRL2 and CCR $3 .{ }^{\circ} \mathrm{We}$ therefore determined whether the frequency of CCRL2 and CCR3 surface marker expression was different in patients with ALS and healthy control patients in $\mathrm{CD}_{16}{ }^{+}$and $\mathrm{CD}^{-} 6^{-}$monocyte populations. We observed that in $\mathrm{CD}_{1} 6^{+}$monocytes, there was a significant increase in the percentage of cells expressing CCRL2, but there was no difference in the total number of expressing cells (figure 3A). Similarly, we observed no changes in the expression pattern of CCRL2 in CD16- monocytes.

In contrast, CCR3 expression was altered in $\mathrm{CD}_{16}{ }^{-}$monocytes rather than $\mathrm{CD} 16^{+}$monocytes: $\mathrm{CD}_{16}{ }^{-}$monocytes displayed a significantly reduced percentage of cells expressing CCR3 as well as a significantly reduced number of CCR3-positive $\mathrm{CD}^{-} 6^{-}$monocytes (figure 3B). However, when data for both $\mathrm{CD}_{1} 6^{+}$and 


\section{A. $\%$ of leukocytes}

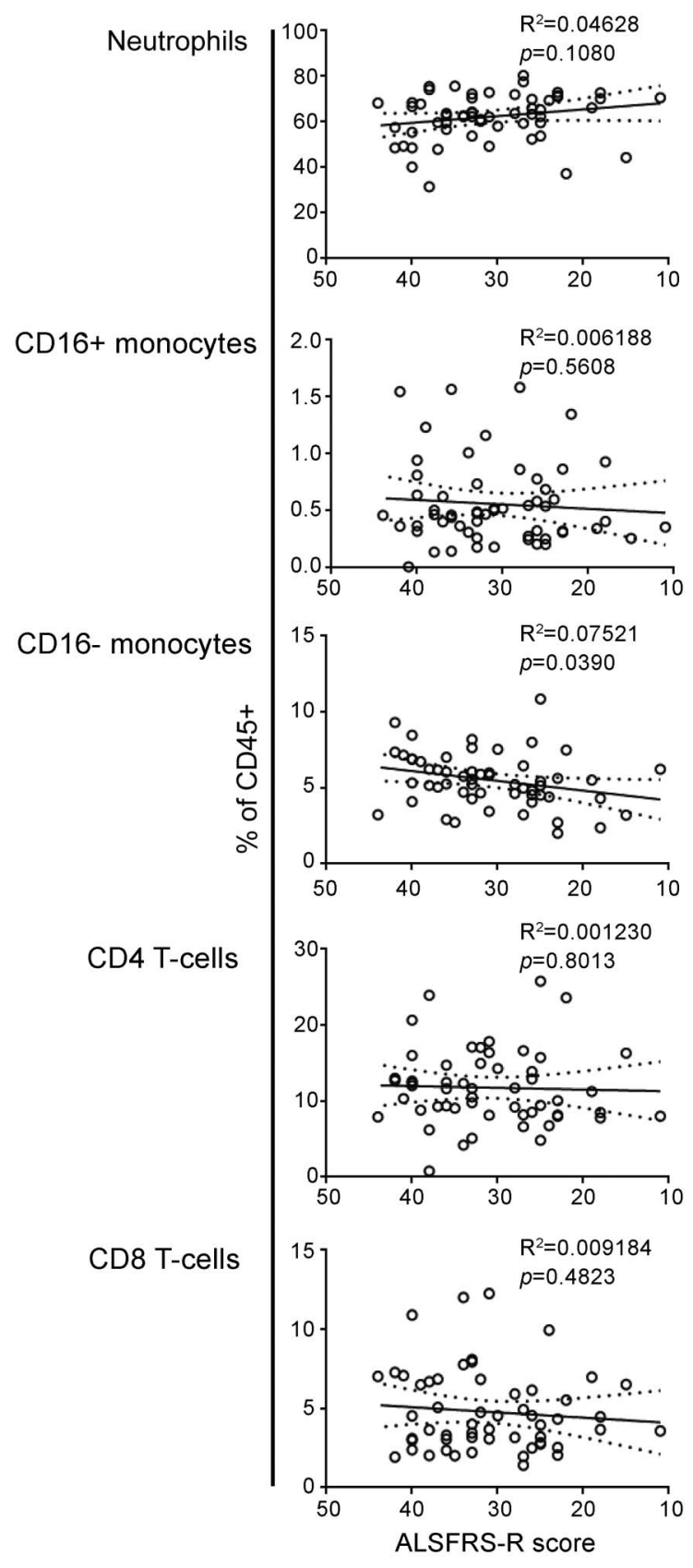

B. \# of leukocytes

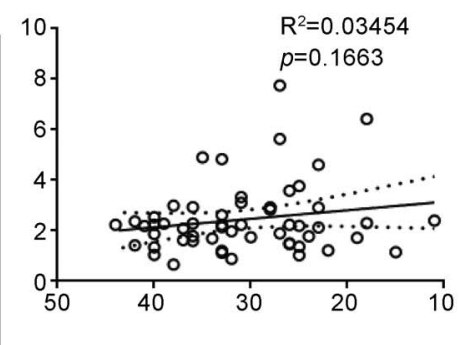

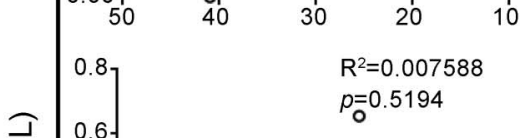
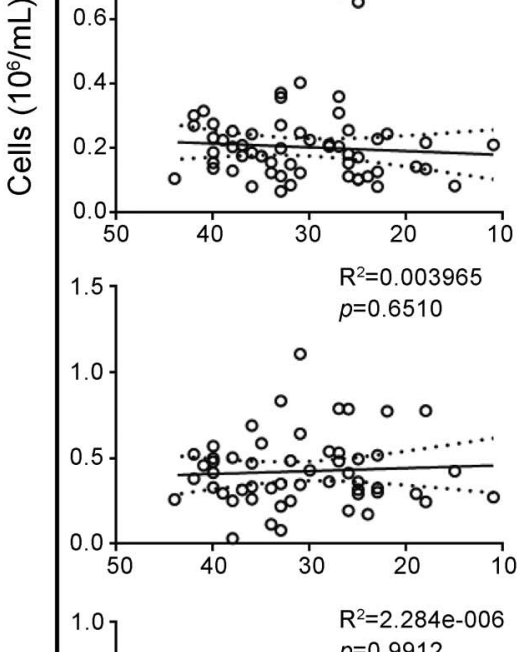

$\circ$

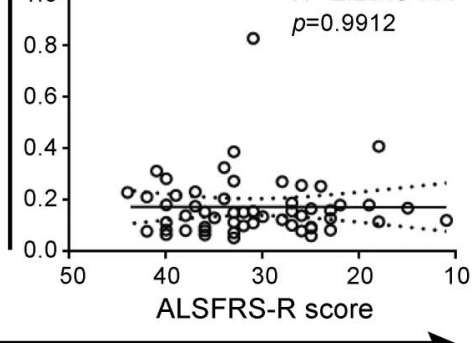

Disease progression

The percentage and total number of leukocytes in the blood of patients with amyotrophic lateral sclerosis are correlated with their ALSFRS-R score at the time of blood draw. Data are displayed with best-fit line and $95 \%$ confidence intervals. $R^{2}$ value and $p$ value are also shown. ALSFRS-R scores are displayed from high (A) to low (B) to illustrate disease progression. ALSFRS-R = Amyotrophic Lateral Sclerosis Functional Rating Scale-Revised.

$\mathrm{CD}^{-} 6^{-}$monocytes were correlated with parameters of disease, we observed no significant correlation between CCR3 or CCRL2 surface expression and ALSFRS-R, duration of disease, progression rate, or site of onset (data not shown). These data indicate that changes in $\mathrm{CD}_{16}{ }^{-}$ monocyte CCR3 expression are not significantly affected by the disease stage.
$\mathrm{N}: \mathrm{M}$ ratio correlates with disease progression. To better correlate immune cell populations with ALS progression, we examined 2 separate cell populations simultaneously. Having observed an increase in neutrophils during disease while observing a decrease in $\mathrm{CD}^{-} 6^{-}$ monocytes, we calculated the N:M ratio for each individual patient. We observed that the N:M ratio 
Figure 3 Percentage and total number of $\mathrm{CD}^{1} 6^{-}$monocytes expressing CCR3 is reduced in patients with ALS

A. CCRL2
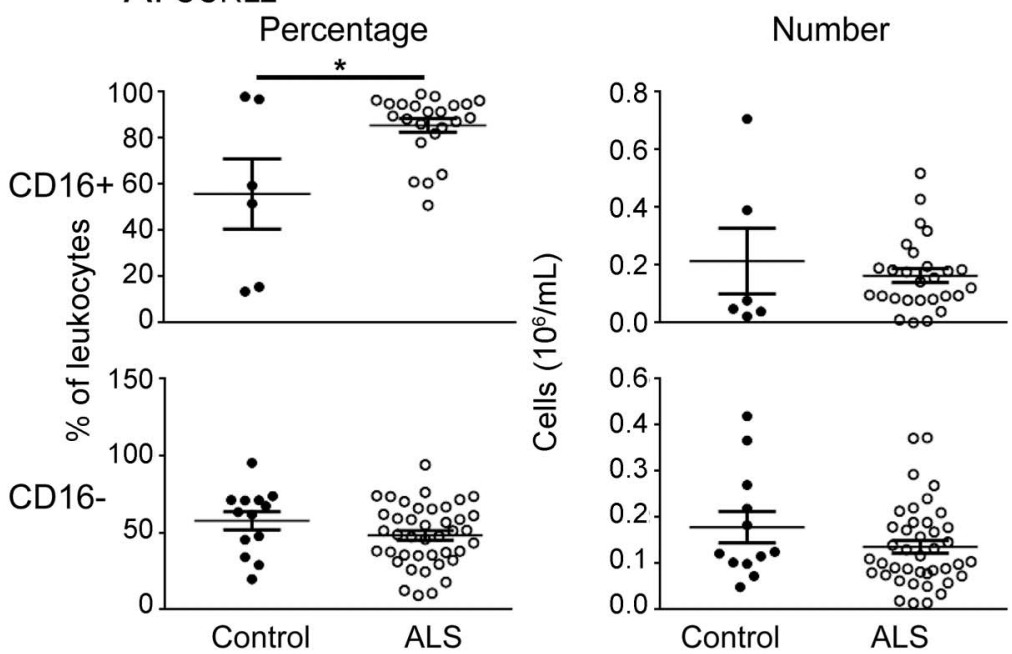

B. $\mathrm{CCR} 3$
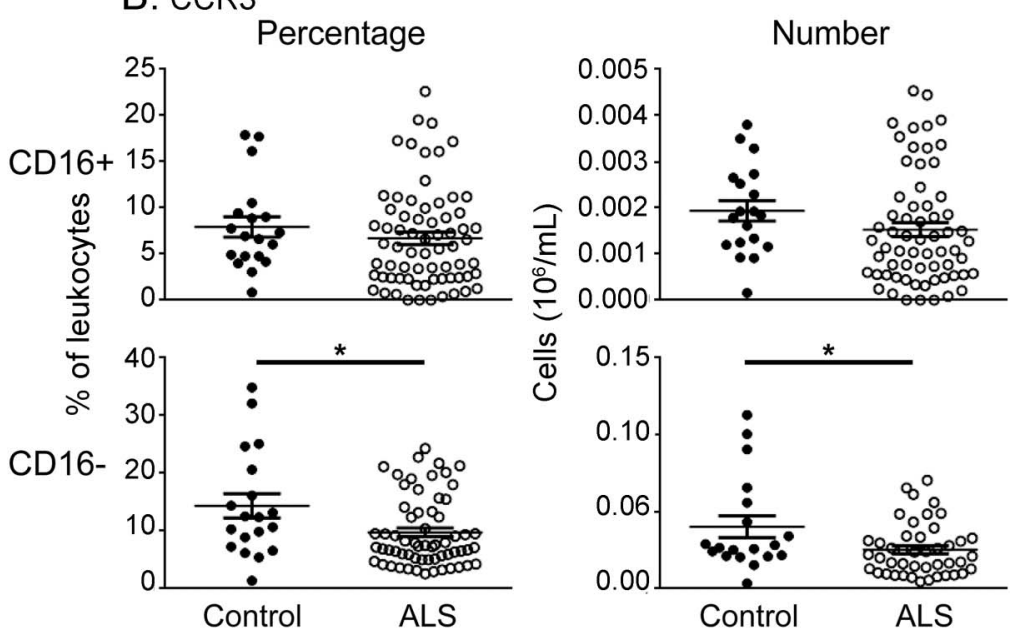

The percentage and total number of $\mathrm{CD}_{1} 6^{+}$and $\mathrm{CD} 16^{-}$monocytes expressing CCRL2 (A) and CCR3 (B) in the peripheral blood are shown. Filled black circles represent individual healthy human control patients, and empty circles represent patients with ALS. Average and SEM are displayed. Asterisks above bars indicate significance $(* p<0.025)$. ALS $=$ amyotrophic lateral sclerosis. was significantly elevated in patients with ALS compared to healthy control patients; the $\mathrm{N}: \mathrm{M}$ ratio increased nearly $50 \%$ (figure 4A). Even more striking, when correlated with ALSFRS-R score, there was a highly significant relationship between disease score and the N:M ratio: as the ALSFRS-R score dropped (and disease became more severe), the $\mathrm{N}: \mathrm{M}$ ratio significantly increased (figure $4 \mathrm{~B}$ ). Finally, the N:M ratio was significantly linked only to ALSFRS-R score, as other metrics of disease such as progression rate and disease duration were not significantly correlated (data not shown). This indicates that the N:M ratio is a remarkable predictor of ALS disease state that is not significantly altered by outside variables.
DISCUSSION The present study examined the role of blood-borne leukocytes and their role in driving ALS. More specifically, we attempted to correlate leukocyte numbers and their surface marker expression with clinical metrics of disease in order to identify potential biomarkers and mechanisms of disease that will ultimately lead to new and effective therapies. We obtained peripheral blood from patients with ALS and healthy controls and used flow cytometry to assess the total number of leukocytes as well as surface marker expression in specific populations of cells. The analysis of ALS using peripheral blood leukocytes is important for several reasons. First, it is now well established that ALS progression features a key immunologic component. ${ }^{2,3}$ Second, ALS requires multiple cell types to drive disease, as neuronal death alone does not trigger disease pathology ${ }^{9,10}$; immune polarization has a critical role in determining whether the CNS environment is protective or destructive.,11 Third, previous in vivo mouse studies have managed to alter ALS progression by removing specific cell populations in the periphery via antibody depletion or genetic knockout ${ }^{12-15}$; manipulation of the immune system outside the CNS can therefore have an effect on disease pathology. Fourth, analysis and treatment focusing on blood-borne leukocytes is minimally invasive and does not require potentially damaging ingress into the fragile CNS.

Consistent with previous reports, ${ }^{13,16-18}$ we observed an increase in the percentage of neutrophils within the total leukocyte populations. This is important, as neutrophils may have several roles in disease (table e-1 at Neurology.org/nn). Most obviously, they may have a proinflammatory role in driving neurodegeneration and may aid disease pathology by contributing to the breakdown of the brain-spinal cord barrier, ${ }^{19}$ which has been shown to drive ALS. ${ }^{20}$ However, treatment of humans and mouse ALS models with granulocytecolony stimulating factor, which induces granulocytosis, does not exacerbate disease symptoms. ${ }^{21,22}$ An alternate possibility is that neutrophils are playing a role in neuronal repair; neutrophils are some of the first cells to respond and are responsible for initiating the repair response in the CNS. ${ }^{23-25}$ Thus, the observed increase in blood-borne neutrophils may result from the exclusion of these cells following the initiation of the repair response.

We also observed a decrease in the percentage of $\mathrm{CD}_{16}{ }^{-}$monocytes in the peripheral blood of patients with ALS, with reduction correlating with disease progression. A previous study profiling $\mathrm{CD}^{-} 6^{-}$ monocytes in humans and mice showed decreased numbers in the peripheral blood, but accumulation in the CNS of mice. ${ }^{12}$ Therefore, reduced $\mathrm{CD} 16^{-}$ monocytes in the blood of human patients may reflect CNS infiltration: monocytes are found in the CNS 
Figure 4 The N:M ratio is significantly increased in patients with ALS and correlates with disease progression


(A) The N:M ratio in healthy control patients (filled black circles) and patients with ALS (empty circles) is shown. Average and SEM are displayed. Asterisks above bars indicate significance (***p $<0.001$ ). (B) The N:M ratio in the blood of patients with ALS is correlated with the ALSFRS-R score at the time of blood draw. Data are displayed with best-fit line and $95 \%$ confidence intervals. $R^{2}$ value and $p$ value are also shown. ALSFRS-R scores are displayed from high (left) to low (right) to illustrate disease progression. ALS = amyotrophic lateral sclerosis; ALSFRS-R = ALS Functional Rating Scale-Revised; N:M ratio = neutrophil to $\mathrm{CD}_{16}{ }^{-}$monocyte ratio.

near dying neurons in patients with ALS ${ }^{26,27}$ and may promote the activation of microglia. ${ }^{12,28}$ Alternatively, they may suppress the inflammatory response. ${ }^{29}$ Whether these cells are capable of crossing the brain-spinal cord barrier in ALS is still a matter of debate, ${ }^{12,30}$ but it has been shown that depleting inflammatory monocytes in a mouse model of ALS delays disease onset and extends survival. ${ }^{12}$ Of note, whereas another group has observed increased numbers of CD4 $\mathrm{T}$ cells in the peripheral blood, ${ }^{31}$ we observed a significant reduction in the percentage and total number of peripheral CD4 $\mathrm{T}$ cells. This difference is likely attributable to methodology: previous studies examined $\mathrm{CD} 4 \mathrm{~T}$ cells within the mononuclear subfraction of leukocytes, while we examined CD4 $\mathrm{T}$ cells within the entire population of leukocytes. Despite the observed differences, CD4 $\mathrm{T}$ cell levels did not correlate with ALSFRS-R score, indicating that cellular levels were reduced during the entirety of disease, not just an early or late phase. These changes could reflect the recruitment of specific subpopulations of $\mathrm{CD} 4 \mathrm{~T}$ cells, such as regulatory
$\mathrm{T}$ cells or memory $\mathrm{T}$ cells, ${ }^{13}$ as $\mathrm{CD} 4 \mathrm{~T}$ cells have been shown to have a protective role in ALS. ${ }^{14}$

Based on the significant correlation between reduced $\mathrm{CD} 16^{-}$monocyte levels and disease progression, we wished to determine whether surface marker expression on the monocytes of patients with ALS was significantly altered compared to healthy human patients. We focused on expression levels of CCRL2 and CCR3, as we had previously detected that these 2 markers were upregulated in the spinal cord during ALS. ${ }^{8}$ CCLR2 is a chemokine receptor expressed at high levels in activated $\mathrm{T}$ cells, neutrophils, and monocytes that binds the chemoattractant plasma protein chemerin, ${ }^{32}$ which is a potent chemoattractant for immature macrophages and dendritic cells. ${ }^{33}$ It has also been established that CCRL2 expression is upregulated in activated macrophages following both M1- and M2-polarizing forms of stimulation. ${ }^{5,6}$ Similarly, monocytes expressing the CCR3 receptor can bind the chemokine ligand CCL11/eotaxin, which is increased in ALS serum and CSF. ${ }^{34}$ We observed that the percentage of $\mathrm{CD} 6^{+}$monocytes expressing CCRL2 was significantly increased in patients with ALS but that the total number of these cells expressing CCRL2 was not significantly altered. We also observed that the $\mathrm{CD} 16^{-}$monocyte population displayed a reduced percentage expressing CCR3 as well as a reduced total number expressing the surface marker. Therefore, decreased expression of CCR3 on $\mathrm{CD} 16^{-}$inflammatory monocytes may indicate recruitment of cells expressing high levels of this receptor. Further studies will be required to determine the role of CCR3 in ALS pathology.

Despite the changes observed in monocyte surface marker expression, we did not observe a significant correlation between CCR3 or CCRL2 expression in ALS and duration of disease, progression rate, or site of onset. Based on the increased percentage of neutrophils and decreased percentage of $\mathrm{CD} 16^{-}$monocytes observed in patients with ALS, we attempted to establish a correlation between more than 2 cell types and disease progression: for each patient we established a ratio of neutrophils to $\mathrm{CD} 16^{-}$monocytes-the $\mathrm{N}: \mathrm{M}$ ratio- and compared this ratio to the ALSFRS$\mathrm{R}$ score to assess the correlation between neutrophil percentages, monocyte percentages, and disease progression. We found that the N:M ratio was significantly elevated in patients with ALS compared to healthy controls, and that this ratio significantly increased as the ALSFRS-R score dropped and disease worsened. Moreover, other metrics of disease-including duration of disease- did not significantly alter the $\mathrm{N}: \mathrm{M}$ ratio. The observed increase is likely attributable to a higher rate of production in the short-lived neutrophil population coupled with monocyte depletion resulting from recruitment (figure e-1). In addition, the 
increased level of neutrophils late in disease suggests that neutrophils are playing a pathogenic role during disease rather than participating in the repair mechanism. ${ }^{35}$ The N:M ratio therefore represents a potential biomarker of ALS. Further studies will be required to assess the potential utility of this new metric.

Overall, our results show that changes in the number and surface marker expression of peripheral leukocytes are associated with ALS disease progression. These data support previous studies suggesting that immune cells in the blood affect the immune response in the CNS as well as subsequent neurodegeneration, ${ }^{12-15}$ although differences in the sex composition of the control and ALS patient groups may have an effect as well. However, the role of immune cells in ALS may be similar in all patients, as we observed no significant difference in cellular percentage, numbers, or ratio based on patient age or site of disease onset (data not shown). The identified $\mathrm{CCR}^{+}$subset of $\mathrm{CD}^{-} 6^{-}$monocytes that is increased in ALS is worthy of further investigation as a potential disease biomarker. Similarly, the increased neutrophil and decreased monocyte frequencies represented as an N:M ratio in patients with ALS suggest that progressive alterations in peripheral myeloid populations may contribute to functional disease-related changes. Further evaluation and consideration of the N:M ratio as a potential prognostic indicator may prove useful in diagnosis and prognosis of disease. In particular, long-term longitudinal studies of individual patients over time will allow us to further assess the role of the immune system in ALS.

\section{AUTHOR CONTRIBUTIONS}

B.J.M. and D.E.B. designed the study, acquired and analyzed the data, and wrote the manuscript. S.R.K. acquired and analyzed the data. C.F.-R. and C.B helped design the study, acquired data, and critically reviewed the manuscript. B.C.C. and S.A.G. collected clinical outcome data, supervised the statistical analyses, and revised the manuscript. E.L.F. designed and funded the study and revised the manuscript.

\section{ACKNOWLEDGMENT}

The authors thank Andrea Smith, Blake Swihart, Crystal Pacut, and Laura Wyatt for technical support, Dr. Stacey Sakowski for editing assistance, and Drs. Benjamin Segal and David Irani for their scientific advising and equipment support (all at the University of Michigan).

\section{STUDY FUNDING}

Supported by the University of Michigan Program for Neurology Research \& Discovery, the A. Alfred Taubman Medical Research Institute, and the NIH (NS007222 postdoctoral fellowship to D.E.B.).

\section{DISCLOSURE}

B.J. Murdock served as a temporary grant writer for the American Neurological Association, received research support from CReATe Consortium. D.E. Bender and S.R. Kashlan report no disclosures. C. Figueroa-Romero received research support from Katherine Rayner Fund, A. Alfred Taubman Medical Research Institute. C. Backus holds a patent for Trk C antibody, produced at UCSF. B.C. Callaghan consulted and was on the advisory board for PCORI grant, received travel funding from the American Academy of Neurology, World Federation of Neurology, consulted for Advance
Medical, ALS Association, certify Centers, received honoraria from British Medical Journal, received research support from Impeto Medical Inc., NIH. S.A. Goutman received research support from Neuralstem, CDC/ATSDR. E.L. Feldman received research support from Centers for Disease Control, NIH, National Institute of Neurological Disorders and Stroke, NIDDK, Novo Nordisk Foundation, Juvenile Diabetes Research Foundation, USIsrael Binational Science Foundation, American Diabetes Association. Go to Neurology.org/nn for full disclosure forms.

Received February 1, 2016. Accepted in final form March 24, 2016.

\section{REFERENCES}

1. Hooten KG, Beers DR, Zhao W, Appel SH. Protective and toxic neuroinflammation in amyotrophic lateral sclerosis. Neurotherapeutics 2015;12:364-375.

2. Zhao W, Beers DR, Appel SH. Immune-mediated mechanisms in the pathoprogression of amyotrophic lateral sclerosis. J Neuroimmune Pharmacol 2013;8:888-899.

3. Murdock BJ, Bender DE, Segal BM, Feldman EL. The dual roles of immunity in ALS: injury overrides protection. Neurobiol Dis 2015;77:1-12.

4. Wainwright DA, Xin J, Mesnard NA, et al. Exacerbation of facial motoneuron loss after facial nerve axotomy in CCR3-deficient mice. ASN Neuro 2009;1:e00024.

5. Brouwer N, Zuurman MW, Wei T, Ransohoff RM, Boddeke HW, Biber K. Induction of glial L-CCR mRNA expression in spinal cord and brain in experimental autoimmune encephalomyelitis. Glia 2004;46:84-94.

6. Oostendorp J, Hylkema MN, Luinge M, et al. Localization and enhanced mRNA expression of the orphan chemokine receptor L-CCR in the lung in a murine model of ovalbumin-induced airway inflammation. J Histochem Cytochem 2004;52:401-410.

7. Brooks BR, Miller RG, Swash M, Munsat TL; World Federation of Neurology Research Group on Motor Neuron Diseases. El Escorial revisited: revised criteria for the diagnosis of amyotrophic lateral sclerosis. Amyotroph Lateral Scler Other Motor Neuron Disord 2000;1:293-299.

8. Figueroa-Romero C, Hur J, Bender DE, et al. Identification of epigenetically altered genes in sporadic amyotrophic lateral sclerosis. PLoS One 2012;7:e52672.

9. Lino MM, Schneider C, Caroni P. Accumulation of SOD1 mutants in postnatal motoneurons does not cause motoneuron pathology or motoneuron disease. J Neurosci 2002;22: 4825-4832.

10. Pramatarova A, Laganiere J, Roussel J, Brisebois K, Rouleau GA. Neuron-specific expression of mutant superoxide dismutase 1 in transgenic mice does not lead to motor impairment. J Neurosci 2001;21:3369-3374.

11. Deshpande P, King IL, Segal BM. Cutting edge: CNS $\mathrm{CD} 11 \mathrm{c}+$ cells from mice with encephalomyelitis polarize Th17 cells and support CD25+CD4+ T cell-mediated immunosuppression, suggesting dual roles in the disease process. J Immunol 2007;178:6695-6699.

12. Butovsky O, Siddiqui S, Gabriely G, et al. Modulating inflammatory monocytes with a unique microRNA gene signature ameliorates murine ALS. J Clin Invest 2012;122:3063-3087.

13. Banerjee R, Mosley RL, Reynolds AD, et al. Adaptive immune neuroprotection in G93A-SOD1 amyotrophic lateral sclerosis mice. PLoS One 2008;3:e2740.

14. Beers DR, Henkel JS, Zhao W, Wang J, Appel SH. CD4+ T cells support glial neuroprotection, slow disease progression, and modify glial morphology in an animal model of inherited ALS. Proc Natl Acad Sci USA 2008; 105:15558-15563. 
15. Chiu IM, Chen A, Zheng Y, et al. T lymphocytes potentiate endogenous neuroprotective inflammation in a mouse model of ALS. Proc Natl Acad Sci USA 2008;105: 17913-17918.

16. Chio A, Calvo A, Bovio G, et al. Amyotrophic lateral sclerosis outcome measures and the role of albumin and creatinine: a population-based study. JAMA Neurol 2014; 71:1134-1142.

17. Keizman D, Rogowski O, Berliner S, et al. Low-grade systemic inflammation in patients with amyotrophic lateral sclerosis. Acta Neurol Scand 2009;119:383-389.

18. Desport JC, Preux PM, Magy L, et al. Factors correlated with hypermetabolism in patients with amyotrophic lateral sclerosis. Am J Clin Nutr 2001;74:328-334.

19. Weiss SJ. Tissue destruction by neutrophils. N Engl J Med 1989;320:365-376.

20. Garbuzova-Davis S, Sanberg PR. Blood-CNS barrier impairment in ALS patients versus an animal model. Front Cell Neurosci 2014;8:21.

21. Chio A, Mora G, La Bella V, et al. Repeated courses of granulocyte colony-stimulating factor in amyotrophic lateral sclerosis: clinical and biological results from a prospective multicenter study. Muscle Nerve 2011; 43:189-195.

22. Henriques A, Pitzer C, Dittgen T, Klugmann M, Dupuis L, Schneider A. CNS-targeted viral delivery of G-CSF in an animal model for ALS: improved efficacy and preservation of the neuromuscular unit. Mol Ther 2011;19:284-292.

23. Butterfield TA, Best TM, Merrick MA. The dual roles of neutrophils and macrophages in inflammation: a critical balance between tissue damage and repair. J Athl Train 2006; 41:457-465.

24. Kim CF, Moalem-Taylor G. Detailed characterization of neuro-immune responses following neuropathic injury in mice. Brain Res 2011;1405:95-108.

25. Kurimoto T, Yin Y, Habboub G, et al. Neutrophils express oncomodulin and promote optic nerve regeneration. J Neurosci 2013;33:14816-14824.
26. Appel SH, Smith RG, Engelhardt JI, Stefani E. Evidence for autoimmunity in amyotrophic lateral sclerosis. J Neurol Sci 1993;118:169-174.

27. Henkel JS, Engelhardt JI, Siklos L, et al. Presence of dendritic cells, MCP-1, and activated microglia/macrophages in amyotrophic lateral sclerosis spinal cord tissue. Ann Neurol 2004;55:221-235.

28. Beers DR, Henkel JS, Xiao Q, et al. Wild-type microglia extend survival in PU.1 knockout mice with familial amyotrophic lateral sclerosis. Proc Natl Acad Sci USA 2006;103:16021-16026.

29. Vaknin I, Kunis G, Miller O, et al. Excess circulating alternatively activated myeloid (M2) cells accelerate ALS progression while inhibiting experimental autoimmune encephalomyelitis. PLoS One 2011;6:e26921.

30. Ajami B, Bennett JL, Krieger C, Tetzlaff W, Rossi FM. Local self-renewal can sustain CNS microglia maintenance and function throughout adult life. Nat Neurosci 2007; 10:1538-1543.

31. Mantovani S, Garbelli S, Pasini A, et al. Immune system alterations in sporadic amyotrophic lateral sclerosis patients suggest an ongoing neuroinflammatory process. J Neuroimmunol 2009;210:73-79.

32. Zabel BA, Nakae S, Zuniga L, et al. Mast cell-expressed orphan receptor CCRL2 binds chemerin and is required for optimal induction of $\mathrm{IgE}$-mediated passive cutaneous anaphylaxis. J Exp Med 2008;205:2207-2220.

33. Wittamer V, Franssen JD, Vulcano M, et al. Specific recruitment of antigen-presenting cells by chemerin, a novel processed ligand from human inflammatory fluids. J Exp Med 2003;198:977-985.

34. Kuhle J, Lindberg RL, Regeniter A, et al. Increased levels of inflammatory chemokines in amyotrophic lateral sclerosis. Eur J Neurol 2009;16:771-774.

35. Pineau I, Sun L, Bastien D, Lacroix S. Astrocytes initiate inflammation in the injured mouse spinal cord by promoting the entry of neutrophils and inflammatory monocytes in an IL-1 receptor/MyD88-dependent fashion. Brain Behav Immun 2010;24:540-553. 


\section{Neurology \\ Neuroimmunology \& Neuroinflammation}

Increased ratio of circulating neutrophils to monocytes in amyotrophic lateral sclerosis

Benjamin J. Murdock, Diane E. Bender, Samy R. Kashlan, et al.

Neurol Neuroimmunol Neuroinflamm 2016;3;

DOI 10.1212/NXI.0000000000000242

This information is current as of June 1, 2016

Updated Information \&

Services

Supplementary Material

References

Citations

Subspecialty Collections

Permissions \& Licensing

Reprints including high resolution figures, can be found at:

http://nn.neurology.org/content/3/4/e242.full.html

Supplementary material can be found at:

http://nn.neurology.org/content/suppl/2016/06/01/3.4.e242.DC1

This article cites 35 articles, 10 of which you can access for free at: http://nn.neurology.org/content/3/4/e242.full.html\#\#ref-list-1

This article has been cited by 5 HighWire-hosted articles: http://nn.neurology.org/content/3/4/e242.full.html\#\#otherarticles

This article, along with others on similar topics, appears in the following collection(s):

All Immunology

http://nn.neurology.org//cgi/collection/all_immunology

Amyotrophic lateral sclerosis

http://nn.neurology.org//cgi/collection/amyotrophic_lateral_sclerosis_

Information about reproducing this article in parts (figures,tables) or in its entirety can be found online at:

http://nn.neurology.org/misc/about.xhtml\#permissions

Information about ordering reprints can be found online:

http://nn.neurology.org/misc/addir.xhtml\#reprintsus

Neurol Neuroimmunol Neuroinflamm is an official journal of the American Academy of Neurology.

Published since April 2014, it is an open-access, online-only, continuous publication journal. Copyright $\odot$ 2016 American Academy of Neurology. All rights reserved. Online ISSN: 2332-7812.

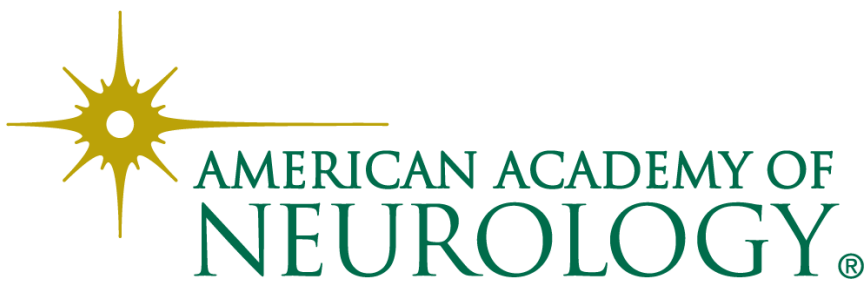

